\title{
Logistics network design
}

\author{
Stefan Nickel • Francisco Saldanha-da-Gama
}

Published online: 13 February 2009

(C) Springer-Verlag 2009

In the last decade, logistics network design has attracted the attention of many researchers. The strategic nature of many network design decisions together with the need to integrate them with tactical and operational decisions explains why the topic has become of common interest for communities such as those working in Logistics, Transportation and Location Theory. The importance of the topic has been also recognized by the European Federation of the Operational Research Societies (EURO) when promoting a EURO Winter Institute on Location and Logistics, which was held in Estoril, Portugal, in January 2007. This event was a privileged forum for discussing relevant issues, models and techniques in logistics network design.

In order to collect a representative set of works in the topic, the idea of a focused issue in OR Spectrum was born and was positively supported by the former and current Managing Editors, Hans-Otto Günther and Stefan Minner, respectively.

The call for papers resulted in the submission of 15 papers covering different aspects in the topic. The peer-review process resulted in eight accepted papers. Criteria in the refereeing process have been the originality of the work, the relevance and applicability of the problem studied, the technical quality of the paper and the quality of the results achieved. It should be emphasized that not all the 24 laureates in the EURO Winter Institute on Location and Logistics were encouraged to submit a paper to this

\footnotetext{
S. Nickel $(\varangle)$

Lehrstuhl fur Operations Research und Logistik, Universitat des Saarlandes, Postfach 151150, Geb. A5 3, 66041 Saarbrücken, Germany

e-mail: s.nickel@orl.uni-saarland.de

F. Saldanha-da-Gama

Departamento de Estatística e Investigação Operacional, Faculdade de Ciências da Universidade de Lisboa,

Bloco C6, Piso 4, 1749-016 Lisbon, Portugal

e-mail: fsgama@fc.ul.pt
} 
special issue. Moreover, the participation on this Institute was regulated by a reviewing process. These two facts explain the high quality of the submissions to this feature issue.

The paper by Bruno, Improta and Sgalambro, deals with the problem of finding the best schedule for a set of departing lines in a public transit terminal. Two models are proposed and discussed. The goal is to balance the operation costs of the transportation lines and the waiting time of customers at the terminal. A general solver is used to solve the problem. In the work by Contreras, Días and Fernández the capacitated hub location problem with single assignment is studied. A lagrangian relaxation is proposed for obtaining tight lower and upper bounds. The paper represents an important breakthrough in the resolution of the problem because very small gaps are obtained. Kauder proposes a mixed-integer programming model for the logistic network design problem faced by an international automotive manufacturer. The basic model includes investment decisions in the supply chain planning. Some additional constraints are also considered, which are intended to increase the performance of the model in the event of unexpected factors. A commercial package is considered for solving the proposed model. Özkisacik, Altınel and Aras study a probabilistic extension of the multi-facility Weber problem in which the customer locations are randomly distributed. Heuristics based on the principle of vector quantization are proposed. Plastria and Vanhaverbeke study the inclusion of price decision in the maximal covering model in a competitive environment. Solution approaches are proposed as a consequence of several properties of the problem that are disclosured in this work. Salema, Barbosa-Póvoa and Novais study a logistics network design problem with direct and reverse flows. Strategic network design decisions are integrated with tactical decisions such as production, storage and distribution. The goal is to maximize the profit obtained with the system. A mixed-integer programming model is proposed. To solve the problem the authors proposed the use of a commercial package. Tóth, Plastria, Fernández and Pelegrin study the problem of a chain that wants to locate a new facility in a planar market when a set of facilities is already operating some of which possibly belonging to the chain. A Huff-type customer behavior in a continuous region of the plane is considered. The goal is to maximize the expected profit. This study intends to contribute to a better understanding of the impact of the special pattern and of various parameters of the model on the resulting location and design decisions. Y1ldirmaz, Karabatı and Sayın present a model for determining the optimal lot-sizing and pricing decisions in a single-buyer single-supplier system. An approximate solution procedure is proposed for the problem.

In addition to the previous works, two additional papers had been assigned to this feature issue later on. Doerner, Gutjahr and Nolz propose a multi-criteria approach to the problem of locating public facilities, with particular emphasis to schools, in coastal areas where inundation by tsunamis is a risk to take into account. Three criteria are considered in the model: a measure of the distance between the customers and the facilities, the risk of inundation by a tsunami and the costs for building and maintaining the facilities. Exact and heuristic approaches are proposed and tested in two real-life instances from southern Sri Lanka. Saíz, Hendrix, Fernández and Pelegrin propose a branch-and-bound procedure for the leader problem arising in the context of a Huff-like Stackelberg location problem. The problem arises when two competing 
firms want to locate a new facility in a planar market. Some numerical examples are used to evaluate the performance of the proposed procedure.

The guest editors would like to express their gratitude to the former and current Managing Editors, who provided with OR Spectrum an excellent forum to present a set of contributions in the topic of logistics network design. A word of gratitude is also due to all the referees of the papers submitted to this feature issue. Last but not least, the guest editors would like to thank to Alfredo Marín (University of Murcia, Spain), Ioannis Giannikos (University of Patras, Greece) and Malick Ndiaye (King Fahd University of Petroleum and Minerals, Saudi Arabia) for their assistance during the refereeing process. 